\title{
Article \\ Vitamin D Supplementation in Exclusively Breastfed Infants Is Associated with Alterations in the Fecal Microbiome
}

\author{
Tengfei Ma ${ }^{1}$, Sihan Bu ${ }^{2}$, Nigel Paneth ${ }^{1,3}$, Jean M. Kerver ${ }^{1}$ and Sarah S. Comstock ${ }^{2, *}$ \\ 1 Department of Epidemiology and Biostatistics, College of Human Medicine, Michigan State University, \\ East Lansing, MI 48824, USA; matengfe@msu.edu (T.M.); kerverje@msu.edu (J.M.K.); paneth@msu.edu (N.P.) \\ 2 Department of Food Science and Human Nutrition, Michigan State University, East Lansing, MI 48824, USA; \\ busihan@msu.edu \\ 3 Department of Pediatrics and Human Development, College of Human Medicine, Michigan State University, \\ East Lansing, MI 48824, USA \\ * Correspondence: comsto37@msu.edu
}

check for updates

Citation: Ma, T.; Bu, S.; Paneth, N.; Kerver, J.M.; Comstock, S.S. Vitamin D Supplementation in Exclusively Breastfed Infants Is Associated with Alterations in the Fecal Microbiome. Nutrients 2022, 14, 202. https:// doi.org/10.3390/nu14010202 Academic Editor: Ekhard E. Ziegler Received: 23 November 2021 Accepted: 30 December 2021 Published: 1 January 2022

Publisher's Note: MDPI stays neutral with regard to jurisdictional claims in published maps and institutional affiliations.

Copyright: (c) 2022 by the authors. Licensee MDPI, Basel, Switzerland. This article is an open access article distributed under the terms and conditions of the Creative Commons Attribution (CC BY) license (https:// creativecommons.org/licenses/by/ $4.0 /)$.

\begin{abstract}
Breastfeeding and introduction of solid food are the two major components of infant feeding practices that influence gut microbiota composition in early infancy. However, it is unclear whether additional factors influence the microbiota of infants either exclusively breastfed or not breastfed. We obtained 194 fecal samples from infants at 3-9 months of age, extracted DNA, and sequenced the V4 region of the $16 \mathrm{~S}$ rRNA gene. Feeding practices and clinical information were collected by questionnaire and abstraction of birth certificates. The gut microbiota of infants who were exclusively breastfed displayed significantly lower Shannon diversity ( $p$-adjust $<0.001$ ) and different gut microbiota composition compared to infants who were not breastfed ( $p$-value $=0.001$ ). Among the exclusively breastfed infants, recipients of vitamin D supplements displayed significantly lower Shannon diversity ( $p$-adjust $=0.007$ ), and different gut microbiota composition structure than non-supplemented, breastfed infants ( $p$-value $=0.02$ ). MaAslin analysis identified microbial taxa that associated with breastfeeding and vitamin D supplementation. Breastfeeding and infant vitamin D supplement intake play an important role in shaping infant gut microbiota.
\end{abstract}

Keywords: infant gut microbiota; feeding practices; diet; breastfeeding; vitamin D supplementation; cohort

\section{Introduction}

The gut microbiota has been considered an "invisible organ" of the human body, playing important roles in modulating host functions, including metabolism, digestion, and gut mucosal immune responses and integrity [1,2]. Dysbiosis of the gut microbiota may be associated with various adverse health outcomes in infants such as asthma, Crohn's disease, inflammatory bowel disease, and type 1 diabetes (T1D) [3-7]. The colonization of gut bacteria begins at birth and remains remarkably dynamic until about 2-3 years of age when more stable microbial profiles begin to emerge $[8,9]$. In addition to the mode of delivery and antibiotic exposure, infant feeding practices are key factors in shaping early microbiota composition $[10,11]$. Recent studies have shown that gut microbial profiles in breastfed infants are significantly different from those in formula-fed infants and change rapidly after the transition from breastfeeding to formula or solid food [12-15]. The differences in gut microbiota composition observed between formula-fed and breastfed infants have, at least partially, been attributed to the absence of human milk oligosaccharides (HMOs) in infant formula [16]. Human milk is enriched with HMOs, which have been linked to beneficial bacteria in the gut microbiota $[17,18]$. The introduction of solid food represents another key factor influencing the composition of infant gut microbiota, producing an adult-type complex microbiome dominated by the phyla Bacteroidetes and Firmicutes $[8,19]$. 
Most gut microbiota research on infant nutrition to date has focused on breastfeeding and the introduction of solid food. Little is known about the effect of other dietary features within the two different feeding practices. It is recommended that infants who are breastfed exclusively should take vitamin D supplements every day due to the variability of vitamin D content in human breastmilk [20]. As all infant formula in the United States is fortified with vitamin D, infants who are fed exclusively with formula usually do not need vitamin D supplementation. Vitamin D not only prevents rickets, but also plays an important role in immune responses and metabolic processes that maintain the integrity of the gut epithelium [21-23].

Human milk can also be provided by bottle, from banking of milk by the mother or from human milk banks. This indirect form of breastfeeding can lead to enrichment by environmental bacteria, such as Stenotrophomonas and Pseudomonadacea [16]. The water used to reconstitute powdered infant formulas may also be an important exposure for infant health outcomes. Reconstituting formula with tap water can lead to excessive fluoride and lead intake [24,25]. Different water types (e.g., city water, well water, filtration systems) can be a source of varied bacterial composition.

We sought to determine the association between maternal and infant characteristics and infant feeding practices and the gut microbiota profiles in 3- to 9-month-old infants. In addition, we analyzed the exclusively breastfed and non-breastfed infants separately to assess the impact of unique feeding practices features on the microbiotas of the infants in each group.

\section{Materials and Methods}

\subsection{Study Participants}

The study population was drawn from the Michigan Archive for Research on Child Health (MARCH) cohort [26], an ongoing population-based pregnancy and birth cohort set in Michigan's lower peninsula. The purpose of the MARCH study is to store biological specimens and other health information in pregnancy and early life that can be used to better understand the causes of problems in pregnancy and optimize the health of children. This cohort is a component of a nation-wide study of child health called the Environmental influences on Child Health Outcomes (ECHO) [27]. Our analysis included mothers who provided informed consent for providing infant stool samples. During the MARCH 3 month phone interview mothers confirmed their interest in participating in this sample collection. The fecal Collection kits were assembled at the lab and sent to the participants via mail. The collection kits included instructions for taking a fecal sample, an OMNIgene-GUT tube for sample collection, a box with postage to return the sample, and diapers for the infant sample. Samples were returned to the lab by mail, and fecal aliquots were stored at $-80{ }^{\circ} \mathrm{C}$ upon reaching the lab. 194 fecal samples have so far been collected from singleton infants aged from 3 to 9 months old. The infants in this analysis were 3-9 months of age between 2018 and 2021.

\subsection{Data Collection}

Several questionnaires were administered to mothers from the first prenatal visit through 9 months postpartum. The questionnaire at the first prenatal visit included demographic information about the mothers, their breastfeeding plans and many health-related practices and conditions as well as their estimated due date. Infant dietary feeding patterns, including breast milk and/or formula, detailed feeding practices, and complementary food intake, were collected at the same time as the fecal samples. Detailed information, including the infant's sex, birth weight, complications of pregnancy, mode of delivery (vaginal vs. C-section), pre-pregnancy BMI and gestational age, was abstracted from the birth certificate. 


\subsection{Fecal Microbiota Analysis}

Once received in the lab, the fecal samples were aliquoted into sterile tubes and stored at $-80^{\circ} \mathrm{C}$. DNA was extracted following a modified version of the Human Microbiome Project's protocol as described previously [28]. Barcoded primers were used to amplify the V4 region of the 16S rRNA gene following the mothur wet lab documentation. PCR amplification also followed the wet lab protocol outlined in the mothur documentation. The resulting $16 \mathrm{~S}$ rRNA libraries were sequenced using 250 base pair Illumina MiSeq with V2 chemistry at the Michigan State University genomics core. After trimming, clean sequences were analyzed using the QIIME2 (2021. 2 version) pipeline [29]. Demultiplexed sequences were further quality filtered and clustered using QIIME2's DADA2 plugin to generate the ASV table [30]. A phylogenetic tree was constructed from the sequences using the QIIME2 FastTree plugin with default parameters [31]. Unique amplicon sequence variants (ASVs) were assigned a taxonomy by the QIIME2 feature-classifier plugin, using the Silva 132 database at the similarity threshold of $99 \%$ (for $16 \mathrm{~S}$ data) [32,33]. Samples were rarefied to 6000 sequencing reads per sample, leaving 191 stool samples with 6905 unique ASVs, and findings were summarized at the genus taxonomic level.

\subsection{Statistical Analysis}

We used multivariate ordinal logistic regression models to estimate the association between pre-pregnancy BMI and breastfeeding practices, with adjustment for demographic variables and delivery mode.

Gut microbiota is analyzed by alpha diversity (Chao1 index and Shannon index) and beta diversity (Bray-Curtis dissimilarity and Weighted UniFrac) using the "vegan" package in $R$ [34]. The difference of alpha diversity and relative abundance of taxa between feeding practices groups were tested by Wilcoxon rank test and Kruskal-Wallis test with false discovery rate (FDR) correction for multiple comparisons. We assessed the influence of factors significantly associated with gut bacterial community structure by multivariate models using a Permutational Multivariate Analysis of Variance (PERMANOVA) with 999 permutations based on Bray-Curtis dissimilarities (adonis, $R$ vegan package) $[34,35]$. PERMANOVA is non-parametric multivariate statistical test, with $p$-values obtained using appropriate distribution-free permutation techniques. We used the multivariate association with linear models (MaAsLin) to identify associated microbiological taxa with the feeding practices and other related factors [36,37]. MaAsLin is a multivariate statistical framework that identifies associations between clinical metadata and microbial community abundance and provides both nominal $p$-values and FDR adjusted $p$-values (q-values) by BenjaminiHochberg procedure [38]. Associations are considered significant when the q-value is below the threshold of 0.1 .

\section{Results}

\subsection{Participants and Feeding Practices}

We analyzed gut microbiome samples from 191 infants. In Table 1, maternal and infant characteristics are compared by breastfeeding status (exclusive breastfeeding, partial breastfeeding, and not breastfeeding). During the week immediately preceding stool sample collection, $88(46.1 \%)$ infants were fed exclusively with breast milk, $43(22.5 \%)$ were fed partially with breast milk, and $60(31.4 \%)$ were not fed with breast milk. The median age at the time of specimen collection was 3.8 months (range: 3.0 months -9.3 months). Partially breastfed infants were more likely to be fed with complementary foods than those who were not breastfed $(44.2 \%$ vs. $35.0 \%, p>0.4)$. Infants who were exclusively breastfed were more likely to be given vitamin D supplementation than partially breastfed or not-breastfed infants $(39.8 \%$ vs. $18.6 \%$ vs. $1.7 \%, p<0.01)$. A higher proportion of mothers who practiced exclusive breastfeeding were of normal BMI (18.5-25.0) prior to pregnancy comparing to those who practiced partial breastfeeding or who were not breastfeeding $(50.0 \% \mathrm{vs} .39 .5 \%$ vs. $26.7 \%, p<0.01$ ). Mothers who practiced exclusive breastfeeding were more likely to have a college degree than women who partially breastfed or did not breastfeed $(72.4 \%$ vs. 
$61.9 \%$ vs. $32.2 \%, p<0.01$ ). In a multivariate model adjusted for maternal age, maternal educational level, pre-pregnancy BMI (continuous), delivery mode, and infant age, mothers with higher pre-pregnancy BMI were less likely to practice breastfeeding $(\mathrm{OR}=0.95, \mathrm{CI}$ : $0.91-0.99, p$-value $=0.01$, Table 2 ), and mothers with higher educational level were more likely to practice breastfeeding $(\mathrm{OR}=2.66, \mathrm{CI}: 1.72-4.21, p$-value $<0.001$, Table 2$)$.

Table 1. Characteristics of the mothers and infants by breastfeeding status ${ }^{1}$.

\begin{tabular}{|c|c|c|c|c|}
\hline Characteristic & $\begin{array}{c}\text { Exclusive } \\
\text { Breastfeeding } \\
(\mathrm{N}=88)\end{array}$ & $\begin{array}{l}\text { Partial Breastfeeding } \\
\qquad(\mathrm{N}=43)\end{array}$ & $\begin{array}{l}\text { No Breastfeeding } \\
\quad(N=60)\end{array}$ & $p$-Value \\
\hline $\begin{array}{l}\text { Infant age at sample collection (day), } \\
\text { mean (SD) }\end{array}$ & $115.5(17.3)$ & $135.9(39.8)$ & $126.3(31.9)$ & $<0.01$ \\
\hline $\begin{array}{l}\text { Infant had any antibiotics since birth, } \\
\mathrm{n}(\%)\end{array}$ & $14(15.9)$ & 5 (11.6) & $8(13.3)$ & 0.40 \\
\hline $\begin{array}{l}\text { Consumption of complementary food } \\
\text { during past } 24 \mathrm{~h}, \mathrm{n}(\%)\end{array}$ & $0(0.0)$ & $19(44.2)$ & $21(35.0)$ & $<0.01^{3}$ \\
\hline $\begin{array}{c}\text { Infant probiotic supplement }{ }^{2} \text { during } \\
\text { past } 24 \mathrm{~h}, \mathrm{n}(\%)\end{array}$ & $4(4.5)$ & $1(2.4)$ & $3(3.3)$ & 0.90 \\
\hline $\begin{array}{l}\text { Infant Vitamin D supplement during } \\
\text { past } 24 \mathrm{~h}, \mathrm{n}(\%)\end{array}$ & $35(39.8)$ & $8(18.6)$ & $1(1.7)$ & $<0.01$ \\
\hline \multicolumn{5}{|l|}{ Delivery mode, $\mathrm{n}(\%)$} \\
\hline Vaginal delivery & $66(75)$ & $30(69.8)$ & $36(60)$ & \multirow{2}{*}{0.10} \\
\hline C-section & $22(25)$ & $13(30.2)$ & $24(40)$ & \\
\hline $\begin{array}{l}\text { Infant weight at delivery (gram), } \\
\text { mean (SD) }\end{array}$ & $3461(551)$ & $3336(529)$ & $3269(598)$ & 0.10 \\
\hline \multicolumn{5}{|l|}{ Infant sex, n (\%) } \\
\hline Male & $43(48.9)$ & $22(51.2)$ & $30(50)$ & \multirow{2}{*}{0.98} \\
\hline Female & $45(51.1)$ & $21(48.8)$ & $30(50)$ & \\
\hline \multicolumn{5}{|l|}{ Maternal pre-pregnancy BMI, n (\%) } \\
\hline$<18.5$ & $1(1.1)$ & $0(0.0)$ & $3(5.0)$ & \multirow{4}{*}{$<0.01$} \\
\hline $18.5-25$ & $44(50)$ & $17(39.5)$ & $16(26.7)$ & \\
\hline$>25-30$ & $24(27.3)$ & $11(25.6)$ & $12(20.0)$ & \\
\hline$>30$ & $19(21.6)$ & $15(34.9)$ & $29(48.3)$ & \\
\hline \multicolumn{5}{|l|}{ Maternal education level, n (\%) } \\
\hline Did not finish high school & $0(0.0)$ & $0(0.0)$ & $6(10.2)$ & \multirow{4}{*}{$<0.01$} \\
\hline High school graduate or GED & $4(4.6)$ & $4(9.5)$ & $21(35.6)$ & \\
\hline Some college & $20(23.0)$ & $12(28.6)$ & $13(22.0)$ & \\
\hline College graduate or more & $63(72.4)$ & $26(61.9)$ & $19(32.2)$ & \\
\hline
\end{tabular}

${ }^{1}$ Breastfeeding status information was collected at the time of fecal sample collection. Values are mean (SD) for continuous variables or $\mathrm{n}(\%)$ for categorical variables. Difference by breastfeeding status was calculated using an ANOVA or chi-squared test. ${ }^{2}$ Including probiotic supplement, kefir and kimchi. ${ }^{3}$ Post hoc analysis with Bonferroni adjustment showed significant difference in consumption of complementary food between partial breastfeeding and no breastfeeding groups.

Table 2. Association between breastfeeding (exclusive, partial, and no breastfeeding) and perinatal characteristics ${ }^{1}$.

\begin{tabular}{cccc}
\hline Maternal Characteristics & $\begin{array}{c}\text { Proportional Odds } \\
\text { Ratio }\end{array}$ & $\mathbf{9 5 \% ~ C I ~}$ & $p$-Value \\
\hline Maternal age(year) & 1.02 & $0.96-1.09$ & 0.48 \\
Maternal educational level & 2.66 & $1.72-4.21$ & $<0.001$ \\
Pre-pregnancy BMI (continuous) & 0.95 & $0.91-0.99$ & 0.01 \\
Delivery mode (vaginal vs. & 0.56 & $0.29-1.05$ & 0.07 \\
C-section) & 0.99 & $0.98-1.0$ & 0.07 \\
Infant age (day) &
\end{tabular}

${ }^{1}$ A multivariate ordinal logistic regression analysis was performed to assess the association. Variables in the model include maternal age, maternal education level, pre-pregnancy BMI, delivery mode, and infant age. 


\subsection{Gut Microbiota Analysis}

Fecal samples from infants who were exclusively breastfed displayed lower Shannon diversity than samples from those who were not breastfed (FDR adjusted $p$-value $<0.01$, Figure 1A). Samples from infants who were partially breastfed displayed Shannon diversity intermediate between the other two groups, but not significantly different from either (FDR adjusted $p$-value $=0.9$ ). Chao1 index was not significantly different across the three groups (FDR adjusted $p$-value $=1.0$, Figure 1B). Among the exclusively breastfed, infants who had been given a vitamin $\mathrm{D}$ supplement during the previous $24 \mathrm{~h}$ displayed lower Shannon index (FDR adjusted $p$-value $<0.01$, Figure 1C) and lower Chao1 index (FDR adjusted $p$-value $=0.6$, Figure 1D) than those infants who were not given a vitamin D supplement.
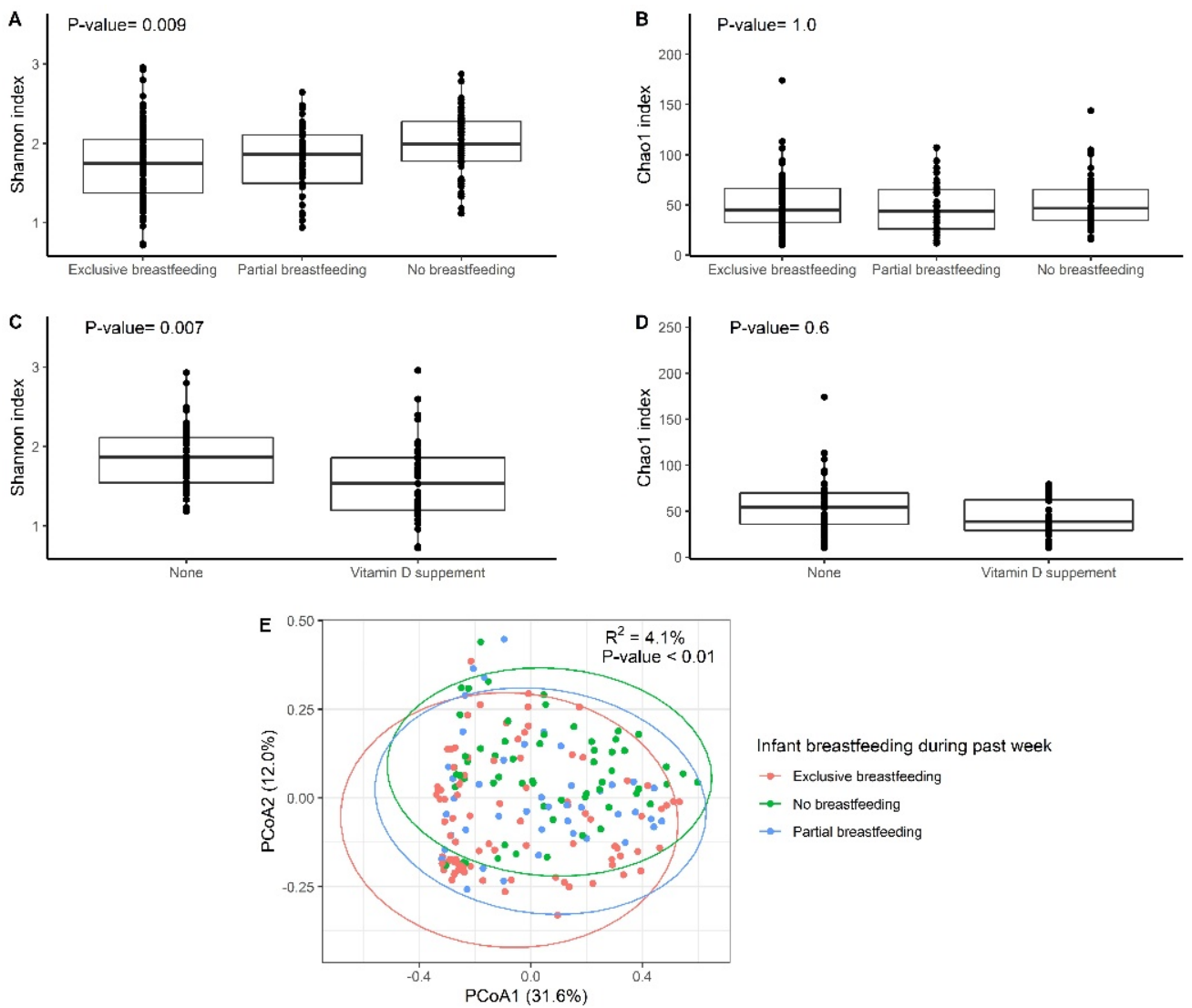

Figure 1. Infant alpha and beta diversity by infant breastfeeding and Vitamin D supplement. FDR adjusted $p$-value for alpha diversity was displayed in upper-left. (A) The Shannon index was used for alpha diversity. All the participants were included in the analysis $(\mathrm{N}=191)$. Group differences were tested by Kruskal-Wallis test. We then performed post hoc test for multiple comparisons. After FDR adjustment, no breastfeeding group has significant difference with exclusive breastfeeding (adjusted $p$-value $<0.01)$. Partial breastfeeding group has no significant difference with exclusive breastfeeding group $(p$-value $=0.4$, adjusted $p$-value $=0.9)$ and no breastfeeding group $(p$-value $=0.03$, adjusted $p$-value $=0.09)$. (B) The Chao1 index was used for alpha diversity. All the participants were included in the analysis $(\mathrm{N}=191)$. Post hoc test did not find any significant difference between groups. (C) The Shannon index was used for alpha diversity. Only breastfeeding participants were included in this subgroup $(\mathrm{N}=92)$. Group differences were tested by Wilcoxon rank test. (D) The Chao1 index was used for alpha diversity. Only breastfeeding participants were included in this subgroup analysis $(\mathrm{N}=92)$. (E) Principal component analysis (PCoA) ordinations of variation based on the Bray-Curtis distance matrix for all infants. $\mathrm{R}^{2}$ and $p$-value were calculated by univariate PERMANOVA test with 999 permutations. 
Whether breast milk was fed directly or was pumped and fed to the infant using a bottle, the Shannon and Chao1 indices of the infant gut microbiota alpha diversity were similar (FDR adjusted $p$-value $=1.0$ and 0.88 , respectively, Figure S1). Among the non-breastfed infants, neither the water type used to reconstitute the formula nor the consumption of complementary food during past $24 \mathrm{~h}$ was associated with gut microbiota alpha diversity as measured by the Shannon or Chao1 indices (Figure S1).

When classified by breastfeeding status, the gut microbiota communities of the infants were well separated in principal coordinate analysis (PCoA) based on the Bray-Curtis distance matrix and the observation was statistically significant as assessed by PERMANOVA $\left(\mathrm{R}^{2}=4.1 \%, p\right.$-value $<0.01$, Figure $\left.1 \mathrm{E}\right)$. In addition to the feeding practices, gestational age $\left(\mathrm{R}^{2}=4.0 \%, p\right.$-value $\left.=0.001\right)$ and delivery mode $\left(\mathrm{R}^{2}=2.0 \%, p\right.$-value $\left.=0.003\right)$ were significantly associated with overall gut microbiome composition (Table 3; multivariate PERMANOVA model on Bray-Curtis distances). The PERMANOVA results were consistent with results from the Weighted UniFrac distance metric (Table S1). We then repeated the PERMANOVA analysis on Bray-Curtis distances within exclusively breastfed and not breastfed infants separately. These subgroup analyses also included additional variables. Accordingly, delivery mode $\left(R^{2}=3.5 \%, p=0.01\right)$ and infant vitamin D supplement in the past $24 \mathrm{~h}\left(\mathrm{R}^{2}=3.4 \%\right.$, $P=0.02$ ) were significantly associated with gut microbiota composition in exclusively breastfed infants (Table S2). Among the not breastfed infants, only maternal education level $\left(\mathrm{R}^{2}=4.1 \%, p\right.$-value $\left.=0.02\right)$ was significantly associated with gut microbiota composition (Table S3). The PERMANOVA results of these two subgroup analyses were consistent with results from the Weighted UniFrac distance metrics (results not shown).

Table 3. Results of Permutational Multivariate Analysis of Variance (PERMANOVA) ${ }^{1}$ with 999 permutations for all infants.

\begin{tabular}{cccc}
\hline Variable & F Value & $\mathbf{R}^{2}$ & $p$-Value \\
\hline Breastfeeding during past week & 4.0 & $4.10 \%$ & $0.001 *$ \\
Gestational age & 2.3 & $1.20 \%$ & $0.03 *$ \\
Infant sex & 1.1 & $0.50 \%$ & 0.36 \\
Delivery mode (vaginal vs. C-section) & 3.6 & $1.80 \%$ & $0.004 *$ \\
infant weight at delivery & 0.7 & $0.30 \%$ & 0.72 \\
Infant probiotic supplement during past 24 h & 1.0 & $0.50 \%$ & 0.38 \\
Infant had any antibiotics since birth & 0.9 & $1.00 \%$ & 0.47 \\
Maternal educational level & 2.7 & $1.30 \%$ & $0.02 *$ \\
Maternal pre-pregnancy BMI (continuous) & 0.37 & $0.20 \%$ & 0.96
\end{tabular}

${ }^{1}$ Bray-Curtis distance was used for the PERMANOVA. ${ }^{*}$ indicates the $p$-value $<0.05$.

We further assessed the association between breastfeeding status and 8 most abundant genera by univariate analysis (Figure S2). These 8 most abundant genera were Bacteroides, Bifidobacterium, Veillonella, Escherichia-Shigella, Ruminococcus gnavus, Clostridium sensu stricto 1, Prevotella, and Lachnoclostridium. Exclusive breastfeeding was significantly associated with a higher relative abundance of Bifidobacterium (FDR adjusted $p$-value $=5 \times 10^{-5}$ ) and a lower relative abundance of Lachnoclostridium (FDR adjusted $p$-value $=5.6 \times 10^{-7}$ ).

MaAsLin results revealed that exclusive breastfeeding was significantly associated with the relative abundance of a set of genera, including Intestinibacter, Flavonifractor, Lachnoclostridium, Clostridium innocuum group, Lactobacillus, Bifidobacterium, etc. (Table 4). Infant age at sample collection and maternal pre-pregnancy BMI were associated with higher relative abundance of Lachnospira and Alistipes, respectively (Table 4). Among exclusively breastfed infants, infants who had taken a vitamin D supplement in the previous $24 \mathrm{~h}$ had a lower relative abundance of Haemophilus (Table 5). Among the not breastfed infants, having taken a probiotic supplement in the past $24 \mathrm{~h}$ was associated with higher relative abundance of uncultured Lachnospiraceae and Faecalitalea (Table 5). Maternal prepregnancy BMI was associated with a higher relative abundance of Alistipes (Table 5). 
Table 4. MaAsLin Analysis Results: Associations of infant feeding practices and gut microbiome taxa at genus level adjusted by covariates in all infants $(\mathrm{N}=191)^{1}$.

\begin{tabular}{cccccc}
\hline Taxonomy at Genus Level & Meta Data Value & Coefficient & N/N Not 0 & $p$-Value & q-Value ${ }^{2}$ \\
\hline Intestinibacter & Exclusive breastfeeding & -0.567 & $191 / 64$ & $3.6 \times 10^{-10}$ & $3.0 \times 10^{-7}$ \\
Flavonifractor & Exclusive breastfeeding & -0.896 & $191 / 145$ & $4.0 \times 10^{-8}$ & $1.7 \times 10^{-5}$ \\
Lachnoclostridium & Exclusive breastfeeding & -0.998 & $191 / 146$ & $1.9 \times 10^{-7}$ & $5.2 \times 10^{-5}$ \\
Clostridium innocuum group & Exclusive breastfeeding & -0.393 & $191 / 44$ & $4.9 \times 10^{-6}$ & $6.8 \times 10^{-4}$ \\
Lactobacillus & Exclusive breastfeeding & 0.680 & $191 / 115$ & $4.3 \times 10^{-6}$ & $6.8 \times 10^{-4}$ \\
Lactococcus & Exclusive breastfeeding & -0.287 & $191 / 29$ & $1.7 \times 10^{-5}$ & $1.7 \times 10^{-3}$ \\
Bifidobacterium & Exclusive breastfeeding & 0.535 & $191 / 186$ & $2.4 \times 10^{-4}$ & 0.018 \\
Eisenbergiella & Exclusive breastfeeding & -0.322 & $191 / 24$ & $2.4 \times 10^{-4}$ & 0.018 \\
Colidextribacter & Exclusive breastfeeding & -0.398 & $191 / 40$ & $3.2 \times 10^{-4}$ & 0.022 \\
Akkermansia & Exclusive breastfeeding & -0.550 & $191 / 124$ & $1.3 \times 10^{-3}$ & 0.066 \\
Uncultured Lachnospiraceae & Exclusive breastfeeding & -0.188 & $191 / 20$ & $1.4 \times 10^{-3}$ & 0.069 \\
Haemophilus & Exclusive breastfeeding & 0.463 & $191 / 141$ & $1.7 \times 10^{-3}$ & 0.073 \\
Staphylococcus & Exclusive breastfeeding & 0.293 & $191 / 56$ & $1.8 \times 10^{-3}$ & 0.073 \\
Incertae_Sedis & Exclusive breastfeeding & -0.358 & $191 / 99$ & $1.6 \times 10^{-3}$ & 0.073 \\
Flavonifractor & Partial breastfeeding & -0.909 & $191 / 145$ & $7.2 \times 10^{-7}$ & $1.5 \times 10^{-4}$ \\
Haemophilus & Partial breastfeeding & 0.740 & $191 / 141$ & $1.3 \times 10^{-5}$ & 0.001 \\
Lachnoclostridium & Partial breastfeeding & -0.860 & $191 / 146$ & $5.8 \times 10^{-5}$ & 0.005 \\
\hline Lactococcus & Partial breastfeeding & -0.258 & $191 / 29$ & $5.6 \times 10^{-4}$ & 0.031 \\
Alistipes & Pre-pregnancy BMI & 0.206 & $191 / 99$ & $4.0 \times 10^{-4}$ & 0.025 \\
Lachnospira & Age at sample collection & 0.171 & $191 / 84$ & $5.7 \times 10^{-4}$ & 0.032 \\
\hline
\end{tabular}

${ }^{1}$ Model was adjusted for infant antibiotic use, sex, infant birth weight, delivery mode, age at fecal sample collection, infant probiotic supplement and pre-pregnancy BMI. Not breastfeeding is the reference for the breastfeeding status in the regression model. ${ }^{2} \mathrm{q}$-value is the FDR (Benjamini-Hochberg) adjusted $p$-value. $\mathrm{q}$-value $<0.1$ for multiple comparisons was considered statistically significant and included in the table.

Table 5. MaAsLin Analysis Results: Associations of infant feeding practices and gut microbiome taxa at genus level. adjusted by covariates within exclusively breastfed and no breastfed infants 1 .

\begin{tabular}{|c|c|c|c|c|c|c|}
\hline Breastfeeding Status & $\begin{array}{l}\text { Taxonomy at } \\
\text { Genus Level }\end{array}$ & Meta Data Value & Coefficient & N/N Not 0 & $p$-Value & q-Value ${ }^{2}$ \\
\hline Exclusively $(\mathrm{N}=88)$ & Haemophilus & $\begin{array}{c}\text { Vitamin D } \\
\text { supplement (Yes) }\end{array}$ & -0.683 & $88 / 74$ & $6.7 \times 10^{-5}$ & 0.058 \\
\hline \multirow{3}{*}{ Not breastfeeding $(\mathrm{N}=60)$} & Faecalitalea & $\begin{array}{c}\text { Probiotic } \\
\text { supplement (Yes) }\end{array}$ & 1.718 & $60 / 9$ & $2.9 \times 10^{-9}$ & $2.4 \times 10^{-6}$ \\
\hline & $\begin{array}{c}\text { Uncultured } \\
\text { Lachnospiraceae }\end{array}$ & $\begin{array}{c}\text { Probiotic } \\
\text { supplement (Yes) }\end{array}$ & 1.269 & $60 / 10$ & $5.1 \times 10^{-5}$ & 0.021 \\
\hline & Alistipes & Pre-pregnancy BMI & 0.431 & $60 / 27$ & $2.6 \times 10^{-4}$ & 0.072 \\
\hline
\end{tabular}

${ }^{1}$ Both Models were adjusted for infant antibiotic use, sex, infant birth weight, delivery mode, age at fecal sample collection, infant probiotic supplement and pre-pregnancy BMI. ${ }^{2}$ q-value is the FDR (Benjamini-Hochberg) adjusted $p$-value. q-value $<0.1$ for multiple comparisons was considered statistically significant and results were included in the table.

\section{Discussion}

Our study was conducted in a population with somewhat higher than average rates of exclusive breastfeeding, with nearly half $(46.1 \%)$ of the infants exclusively breastfed at the median age of 3.8 months. This percentage is higher than in the Infant Feeding Practices Study II in the US (34\% at 3 months) in 2007 [39], while it is close to the percentage in CDC National Immunization Survey in 2018 (46.3\% at 3 month) [40]. Although the American Academy of Pediatrics recommends vitamin D supplementation for all breastfed infants [41], only $39.8 \%$ of the mothers in our study followed this recommendation, a lower frequency than that has been found in Canadian and European cohorts in 2009 and 2014, respectively [42,43]. 
We found that higher maternal education level and lower pre-pregnancy BMI were independently and significantly associated with an increased odds of being exclusively breastfed, consistent with previous studies in developed and developing countries $[39,44,45]$. Breastfeeding initiation and duration are also negatively correlated with high pre-pregnancy BMI $[46,47]$. These associations may be attributed to the physiological factors such as delayed onset of lactogenesis II and imbalances of hormones [48]. Previous studies have showed that maternal obesity can cause the delayed onset of lactogenesis II (DOL) that is associated with mother's confidence that her milk is sufficient for her child [49,50]. As a result, it can lead to lower rates of breastfeeding initiation and early termination of exclusive breastfeeding. The associations between maternal BMI and lactation success have been recently reviewed [51,52].

Our study showed the importance of both breastfeeding and infant vitamin D supplements in shaping infant gut microbiota composition. Breastfeeding is significantly associated with both alpha and beta diversity of infant gut microbiota. We observed that the Shannon diversity of partially breastfed infants was between that of exclusively breastfed infants and not breastfed infants, though somewhat closer to the exclusively breast fed, suggesting a dose-response relationship between breastfeeding and Shannon diversity of infant gut microbiota. These results agree with a previous study, which reported that the composition of gut microbiota from partially breastfed infants are similar to that from exclusively breastfed infants [13]. Among the subgroup analysis of exclusively breastfed infants, the alpha and beta diversity results demonstrated that vitamin D supplementation is associated with infant gut microbiota composition. These results are in good agreement with the study of Lei et al. who investigated the role of vitamin D supplement on gut microbiome from 31 exclusively breastfed infants at 4-months-old [53]. Lei et al. showed that vitamin $\mathrm{D}$ supplementation is associated with both alpha diversity and beta diversity of infant gut microbiome [53]. Animal studies demonstrate that vitamin D plays a critical role in maintaining the integrity of the intestinal mucosal barrier by preserving the integrity of junctions that control mucosal permeability and reduction of pro-inflammatory cytokines such as IL-8 $[23,54,55]$. In addition, studies also found that VDR-mediated signaling inhibits inflammation-induced apoptosis of intestinal epithelial cells [54,56]. As a result of these effects on the intestinal mucosa, vitamin $\mathrm{D}$ acts as an important factor influencing the gut microbiota.

Besides the infant feeding practices, the results herein confirm that gestational age, delivery mode and maternal educational level are also significantly associated with gut microbiome composition. These results are consistent with many previous studies $[10,13,16]$. However, delivery mode is only associated with gut microbiota composition among the exclusively breastfed infants, while no significant association was found in the not breastfed infants. This might be attributed to the fact that C-section delivery can delay lactation initiation [57] and shape the bacterial composition of breast milk [58,59]. Maternal educational level is the only factor that significantly associated with infant gut microbiota composition among the not breastfed infants, whereas it was not significant among the exclusively breastfed infants. This finding suggests that not breastfed infants are more susceptible to socio-economic factors, such as educational level, which is normally connected to offspring diet and nutritional status [60]. Hence, this association among the not breastfed infants might be mediated by the types of solid foods introduced and brands of formula purchased. However, our data set did not allow us to test these associations.

Our study confirmed that Bifidobacterium was enriched in breastfed infants when compared with non-breastfed infants. Lower abundance of Bifidobacterium in infants due to early cessation of breastfeeding could potentially inhibit the interaction of bifidobacterialmediated metabolites with the immune system, leading to higher levels of inflammation $[61,62]$. In contrast, the genus Lachnoclostridium (Lachnospiraceae family) was found to be enriched in the non-breastfed infants when compared with exclusively breastfed or partially breastfed infants. In addition, the genera Eisenbergiella and Lachnospiraceae_uncultured, which also belong to Lachnospiraceae family were found to be enriched in the non-breastfed 
infants by MaAslin. These observations agree with previous studies that lower abundance of Lachnospiraceae is associated with breastfeeding at 3 months of age [63]. The evidence from many studies showed that Lachnospiraceae family or specific genera of Lachnospiraceae may be associated with several inflammatory conditions, such as metabolic syndrome, obesity, diabetes, and liver diseases [64-67]. Thus, our results suggest several possible mechanisms that might explain the beneficial effects of breastfeeding on health outcomes.

Notably, the genus Haemophilus was enriched in the breastfed infants. However, exclusively breastfed infants who had taken a vitamin D supplement in the past $24 \mathrm{~h}$ had a lower relative abundance of Haemophilus compared to those exclusively breastfed infants who were not supplemented. Consistent with our study, Fehr et al. showed that breastmilk may specifically provide Haemophilus to the infant gut [13]. Luthold et al. also demonstrated that Haemophilus was less abundant in the group of highest vitamin $\mathrm{D}$ intake [68], supporting the hypothesis that a reduced immune response in vitamin D deficiency could augment the competitive advantage of Haemophilus and influence the composition of the infant gut microbiome [69].

Our study did not detect any effect of feeding with expressed milk, infant antibiotic intake, and water type for formula on gut microbiome. However, sample sizes were small for many of these comparisons. Therefore, pooling data from multiple cohort studies or analysis in larger cohorts with similar data are necessary to confirm this lack of association. For instance, previous studies demonstrated that infants who had been exposed to antibiotics had decreased abundance of Bifidobacteria and Bacteroides in the infant gut microbiome [70]. In our study, we asked the mothers if the infant had taken any antibiotics since birth, whereas the timing of antibiotics administration was unknown. Hence, it's possible that infant gut microbiome had recovered from the dysbiosis states caused by antibiotics at the time of stool sample collection. The inconsistent results may also be attributed to variations in the antibiotic type, dosage, duration [71].

An important limitation of this study is that only a single stool sample was available for analysis. Although the infant feeding practice information was collected at the same time as the stool sample collection and can demonstrate the impact of short-term exposures on the infant gut microbiota composition, we are unable to determine how these factors contribute to the temporal development of the infant gut microbiome. Another limitation is that we did not collect more detailed information, such as dose of vitamin $\mathrm{D}$, maternal vitamin D status, and timing of antibiotic administration. Future studies would benefit from a longitudinal stool sample collection during infancy and a more detailed infant feeding practices questionnaire that not only collects proximal but also long-term data about infant nutritional exposures.

Supplementary Materials: The following supporting information can be downloaded at: https: / / www.mdpi.com/article/10.3390/nu14010202/s1, Table S1: results of PERMANOVA for all infants on Weighted UniFrac distances, Table S2: results of PERMANOVA for exclusively breastfed infants on Bray-Curtis distances, Table S3: results of PERMANOVA for no breastfed infants on Bray-Curtis distances, Figure S1: infant alpha diversity by different infant feeding practices, and Figure S2: association between breastfeeding status and relative abundance of 8 dominant genera.

Author Contributions: Conceptualization, S.S.C., T.M. and J.M.K.; methodology, N.P., S.S.C. and T.M.; formal analysis, S.B., T.M.; investigation, S.B.; resources, N.P., J.M.K., S.S.C.; data curation, S.B. and T.M.; writing—original draft preparation, T.M.; writing—review and editing, all authors; visualization, T.M.; supervision, S.S.C., N.P.; project administration, J.M.K., N.P., S.S.C.; funding acquisition, S.S.C., N.P., J.M.K., T.M. All authors have read and agreed to the published version of the manuscript. 
Funding: This research was supported by the Office of the Director at the National Institutes of Health under award numbers UG3OD023285 and UH3OD023285 and the Michigan Health Endowment Fund under award numbers G-1608-140432 and R-1605-140007. This research could not have been done without the collaboration of Hurley Medical Center and Hurley Residency Clinic in Flint, MI, Hutzel Medical Center, DMC Center for Obstetrics and Gynecology, and University Health Center in Detroit, MI Munson Hospital and Grand Traverse Women's Clinic in Traverse City, MI, University of Michigan Hospital, Von Voigtlander Women's Center, West Ann Arbor Health Center, and Obstetrics and Gynecology at Briarwood Center in Ann Arbor, MI, St. John's Providence Park, Metro Partners in Women's Health, and Women's Health Consultants in Novi, MI, St. Joseph Mercy Hospital, IHA Canton Obstetrics and Gynecology, and IHA Domino Farms in Ann Arbor, MI, Sinai Grace Hospital, Sinai Grace OB-GYN Women's Health Centers, Northwest Women's Care, and DMC Northwest Women's Care in Detroit, MI, Beaumont Dearborn Hospital and Oakwood OB-GYN Associates in Dearborn, MI, Covenant Hospital, Central Michigan University Health in Saginaw, Mi, SHMG OB/GYN, Grand Rapids Women's Health, and Spectrum Health in Grand Rapids, Mi, Blue Water OB/GYN, NorthPointe OB/GYN, and McLaren Port Huron Hospital in Port Huron, Mi. SB was supported by a graduate research assistantship from the Michigan State University Department of Food Science and Human Nutrition.

Institutional Review Board Statement: The study was conducted according to the guidelines of the Declaration of Helsinki and approved by the Institutional Review Board of Michigan State University (protocol 16-1429) on 26 May 2017.

Informed Consent Statement: Informed consent was obtained from all subjects involved in the study.

Data Availability Statement: The data presented in this study are available on request from the corresponding author. The data are not currently publicly available due to ECHO data sharing policy.

Acknowledgments: First and foremost, we thank all participating families. In addition, we acknowledge the contributions of the MARCH recruitment personnel, MARCH study office staff and the University of Michigan Survey Research Center.

Conflicts of Interest: The authors declare no conflict of interest.

\section{References}

1. Guinane, C.M.; Cotter, P.D. Role of the Gut Microbiota in Health and Chronic Gastrointestinal Disease: Understanding a Hidden Metabolic Organ. Therap. Adv. Gastroenterol. 2013, 6, 295-308. [CrossRef]

2. Kau, A.L.; Ahern, P.P.; Griffin, N.W.; Goodman, A.L.; Gordon, J.I. Human Nutrition, the Gut Microbiome and the Immune System. Nature 2011, 474, 327-336. [CrossRef]

3. Arrieta, M.-C.; Stiemsma, L.T.; Dimitriu, P.A.; Thorson, L.; Russell, S.; Yurist-Doutsch, S.; Kuzeljevic, B.; Gold, M.J.; Britton, H.M.; Lefebvre, D.L.; et al. Early Infancy Microbial and Metabolic Alterations Affect Risk of Childhood Asthma. Sci. Transl. Med. 2015, 7, 307ra152. [CrossRef]

4. $\quad$ Gevers, D.; Kugathasan, S.; Denson, L.A.; Vázquez-Baeza, Y.; Van Treuren, W.; Ren, B.; Schwager, E.; Knights, D.; Song, S.J.; Yassour, M.; et al. The Treatment-Naive Microbiome in New-Onset Crohn's Disease. Cell Host Microbe 2014, 15, 382-392. [CrossRef]

5. Vatanen, T.; Franzosa, E.A.; Schwager, R.; Tripathi, S.; Arthur, T.D.; Vehik, K.; Lernmark, Å.; Hagopian, W.A.; Rewers, M.J.; She, J.-X.; et al. The Human Gut Microbiome in Early-Onset Type 1 Diabetes from the TEDDY Study. Nature 2018, 562, 589-594. [CrossRef]

6. Tilg, H.; Moschen, A.R. Microbiota and Diabetes: An Evolving Relationship. Gut 2014, 63, 1513-1521. [CrossRef] [PubMed]

7. Manichanh, C.; Borruel, N.; Casellas, F.; Guarner, F. The Gut Microbiota in IBD. Nat. Rev. Gastroenterol. Hepatol. 2012, 9, 599-608. [CrossRef] [PubMed]

8. Tanaka, M.; Nakayama, J. Development of the Gut Microbiota in Infancy and Its Impact on Health in Later Life. Allergol. Int. 2017, 66, 515-522. [CrossRef]

9. Rodríguez, J.M.; Murphy, K.; Stanton, C.; Ross, R.P.; Kober, O.I.; Juge, N.; Avershina, E.; Rudi, K.; Narbad, A.; Jenmalm, M.C.; et al. The Composition of the Gut Microbiota throughout Life, with an Emphasis on Early Life. Microb. Ecol. Health Dis. 2015, 26, 26050. [CrossRef] [PubMed]

10. Bokulich, N.A.; Chung, J.; Battaglia, T.; Henderson, N.; Jay, M.; Li, H.; Lieber, A.; Wu, F.; Perez-Perez, G.I.; Chen, Y.; et al. Antibiotics, Birth Mode, and Diet Shape Microbiome Maturation during Early Life. Sci. Transl. Med. 2016, 8, 343ra82. [CrossRef] [PubMed]

11. Cortes-Macías, E.; Selma-Royo, M.; García-Mantrana, I.; Calatayud, M.; González, S.; Martínez-Costa, C.; Collado, M.C. Maternal Diet Shapes the Breast Milk Microbiota Composition and Diversity: Impact of Mode of Delivery and Antibiotic Exposure. J. Nutr. 2021, 151, 330-340. [CrossRef] 
12. Ho, N.T.; Li, F.; Lee-Sarwar, K.A.; Tun, H.M.; Brown, B.P.; Pannaraj, P.S.; Bender, J.M.; Azad, M.B.; Thompson, A.L.; Weiss, S.T.; et al. Meta-Analysis of Effects of Exclusive Breastfeeding on Infant Gut Microbiota across Populations. Nat. Commun. 2018, 9, 4169. [CrossRef]

13. Fehr, K.; Moossavi, S.; Sbihi, H.; Boutin, R.C.T.; Bode, L.; Robertson, B.; Yonemitsu, C.; Field, C.J.; Becker, A.B.; Mandhane, P.J.; et al. Breastmilk Feeding Practices Are Associated with the Co-Occurrence of Bacteria in Mothers' Milk and the Infant Gut: The CHILD Cohort Study. Cell Host Microbe 2020, 28, 285-297.e4. [CrossRef]

14. Haddad, E.N.; Sugino, K.Y.; Kerver, J.M.; Paneth, N.; Comstock, S.S. The Infant Gut Microbiota at 12 Months of Age Is Associated with Human Milk Exposure but Not with Maternal Pre-Pregnancy Body Mass Index or Infant BMI-for-Age z-Scores. Curr. Res. Physiol. 2021, 4, 94-102. [CrossRef]

15. Sugino, K.Y.; Ma, T.; Kerver, J.M.; Paneth, N.; Comstock, S.S. Human Milk Feeding Patterns at 6 Months of Age Are a Major Determinant of Fecal Bacterial Diversity in Infants. J. Hum. Lact 2020, 37, 703-713. [CrossRef] [PubMed]

16. Moossavi, S.; Sepehri, S.; Robertson, B.; Bode, L.; Goruk, S.; Field, C.J.; Lix, L.M.; de Souza, R.J.; Becker, A.B.; Mandhane, P.J.; et al Composition and Variation of the Human Milk Microbiota Are Influenced by Maternal and Early-Life Factors. Cell Host Microbe 2019, 25, 324-335. [CrossRef] [PubMed]

17. Walsh, C.; Lane, J.A.; van Sinderen, D.; Hickey, R.M. Human Milk Oligosaccharides: Shaping the Infant Gut Microbiota and Supporting Health. J. Funct. Foods 2020, 72, 104074. [CrossRef] [PubMed]

18. Lawson, M.A.E.; O’Neill, I.J.; Kujawska, M.; Gowrinadh Javvadi, S.; Wijeyesekera, A.; Flegg, Z.; Chalklen, L.; Hall, L.J. Breast Milk-Derived Human Milk Oligosaccharides Promote Bifidobacterium Interactions within a Single Ecosystem. ISME J. 2020, 14, 635-648. [CrossRef]

19. Stewart, C.J.; Ajami, N.J.; O’Brien, J.L.; Hutchinson, D.S.; Smith, D.P.; Wong, M.C.; Ross, M.C.; Lloyd, R.E.; Doddapaneni, H.; Metcalf, G.A.; et al. Temporal Development of the Gut Microbiome in Early Childhood from the TEDDY Study. Nature 2018, 562, 583-588. [CrossRef]

20. CDC Vitamin D Is Needed to Support Healthy Bone Development. Available online: https://www.cdc.gov/breastfeeding/ breastfeeding-special-circumstances/diet-and-micronutrients/vitamin-d.html (accessed on 27 July 2021).

21. Aranow, C. Vitamin D and the Immune System. J. Investig. Med. 2011, 59, 881-886. [CrossRef]

22. Borges, M.C.; Martini, L.A.; Rogero, M.M. Current Perspectives on Vitamin D, Immune System, and Chronic Diseases. Nutrition 2011, 27, 399-404. [CrossRef]

23. Kong, J.; Zhang, Z.; Musch, M.W.; Ning, G.; Sun, J.; Hart, J.; Bissonnette, M.; Li, Y.C. Novel Role of the Vitamin D Receptor in Maintaining the Integrity of the Intestinal Mucosal Barrier. Am. J. Physiol. Gastrointest. Liver Physiol. 2008, 294, G208-G216. [CrossRef]

24. Till, C.; Green, R.; Flora, D.; Hornung, R.; Martinez-Mier, E.A.; Blazer, M.; Farmus, L.; Ayotte, P.; Muckle, G.; Lanphear, B. Fluoride Exposure from Infant Formula and Child IQ in a Canadian Birth Cohort. Environ. Int. 2020, 134, 105315. [CrossRef]

25. Triantafyllidou, S.; Edwards, M. Lead (Pb) in Tap Water and in Blood: Implications for Lead Exposure in the United States. Crit. Rev. Environ. Sci. Technol. 2012, 42, 1297-1352. [CrossRef]

26. About I CHARM Study. Available online: https://www.epi.msu.edu/charmstudy/about (accessed on 30 September 2021).

27. Paneth, N.; Monk, C. The importance of cohort research starting early in life to understanding child health. Curr. Opin. Pediatr. 2018, 30, 292-296. [CrossRef] [PubMed]

28. Sugino, K.Y.; Paneth, N.; Comstock, S.S. Michigan Cohorts to Determine Associations of Maternal Pre-Pregnancy Body Mass Index with Pregnancy and Infant Gastrointestinal Microbial Communities: Late Pregnancy and Early Infancy. PLoS ONE 2019, 14, e0213733. [CrossRef]

29. Bolyen, E.; Rideout, J.R.; Dillon, M.R.; Bokulich, N.A.; Abnet, C.C.; Al-Ghalith, G.A.; Alexander, H.; Alm, E.J.; Arumugam, M.; Asnicar, F.; et al. Reproducible, Interactive, Scalable and Extensible Microbiome Data Science Using QIIME 2. Nat. Biotechnol. 2019, 37, 852-857. [CrossRef]

30. Callahan, B.J.; McMurdie, P.J.; Rosen, M.J.; Han, A.W.; Johnson, A.J.A.; Holmes, S.P. DADA2: High-Resolution Sample Inference from Illumina Amplicon Data. Nat. Methods 2016, 13, 581-583. [CrossRef] [PubMed]

31. Price, M.N.; Dehal, P.S.; Arkin, A.P. FastTree 2-Approximately Maximum-Likelihood Trees for Large Alignments. PLoS ONE 2010, 5, e9490. [CrossRef]

32. Bokulich, N.A.; Kaehler, B.D.; Rideout, J.R.; Dillon, M.; Bolyen, E.; Knight, R.; Huttley, G.A.; Gregory Caporaso, J. Optimizing Taxonomic Classification of Marker-Gene Amplicon Sequences with QIIME 2's Q2-Feature-Classifier Plugin. Microbiome 2018, 6, 90. [CrossRef] [PubMed]

33. Quast, C.; Pruesse, E.; Yilmaz, P.; Gerken, J.; Schweer, T.; Yarza, P.; Peplies, J.; Glöckner, F.O. The SILVA Ribosomal RNA Gene Database Project: Improved Data Processing and Web-Based Tools. Nucleic Acids Res. 2013, 41, D590-D596. [CrossRef]

34. Dixon, P. VEGAN, a Package of R Functions for Community Ecology. J. Veg. Sci. 2003, 14, 927-930. [CrossRef]

35. Anderson, M.J. A New Method for Non-Parametric Multivariate Analysis of Variance. Austral Ecol. 2001, 26, 32-46. [CrossRef]

36. Morgan, X.C.; Tickle, T.L.; Sokol, H.; Gevers, D.; Devaney, K.L.; Ward, D.V.; Reyes, J.A.; Shah, S.A.; LeLeiko, N.; Snapper, S.B.; et al. Dysfunction of the Intestinal Microbiome in Inflammatory Bowel Disease and Treatment. Genome Biol. 2012, 13, R79. [CrossRef]

37. Mallick, H.; Rahnavard, A.; McIver, L.J.; Ma, S.; Zhang, Y.; Nguyen, L.H.; Tickle, T.L.; Weingart, G.; Ren, B.; Schwager, E.H.; et al Multivariable Association Discovery in Population-Scale Meta-Omics Studies. PLOS Computational Biology 2021, 17, e1009442. [CrossRef] 
38. Thissen, D.; Steinberg, L.; Kuang, D. Quick and Easy Implementation of the Benjamini-Hochberg Procedure for Controlling the False Positive Rate in Multiple Comparisons. J. Educ. Behav. Stat. 2002, 27, 77-83. [CrossRef]

39. Nnebe-Agumadu, U.H.; Racine, E.F.; Laditka, S.B.; Coffman, M.J. Associations between Perceived Value of Exclusive Breastfeeding among Pregnant Women in the United States and Exclusive Breastfeeding to Three and Six Months Postpartum: A Prospective Study. Int. Breastfeed. J. 2016, 11, 8. [CrossRef] [PubMed]

40. CDC Results: Breastfeeding Rates. Available online: https://www.cdc.gov/breastfeeding/data/nis_data/results.html (accessed on 1 October 2021).

41. Gartner, L.M.; Greer, F.R.; Section on Breastfeeding and Committee on Nutrition. American Academy of Pediatrics Prevention of Rickets and Vitamin D Deficiency: New Guidelines for Vitamin D Intake. Pediatrics 2003, 111, 908-910. [CrossRef] [PubMed]

42. Uday, S.; Kongjonaj, A.; Aguiar, M.; Tulchinsky, T.; Högler, W. Variations in Infant and Childhood Vitamin D Supplementation Programmes across Europe and Factors Influencing Adherence. Endocr. Connect. 2017, 6, 667-675. [CrossRef] [PubMed]

43. Aghajafari, F.; Field, C.J.; Weinberg, A.R.; Letourneau, N.; APrON Study Team. Both Mother and Infant Require a Vitamin D Supplement to Ensure That Infants' Vitamin D Status Meets Current Guidelines. Nutrients 2018, 10, 429. [CrossRef] [PubMed]

44. Flores, T.R.; Mielke, G.I.; Wendt, A.; Nunes, B.P.; Bertoldi, A.D. Prepregnancy Weight Excess and Cessation of Exclusive Breastfeeding: A Systematic Review and Meta-Analysis. Eur. J. Clin. Nutr. 2018, 72, 480-488. [CrossRef]

45. Tao, X.-Y.; Huang, K.; Yan, S.-Q.; Zuo, A.-Z.; Tao, R.-W.; Cao, H.; Gu, C.-L.; Tao, F.-B. Pre-Pregnancy BMI, Gestational Weight Gain and Breast-Feeding: A Cohort Study in China. Public Health Nutr. 2017, 20, 1001-1008. [CrossRef]

46. Thompson, L.A.; Zhang, S.; Black, E.; Das, R.; Ryngaert, M.; Sullivan, S.; Roth, J. The Association of Maternal Pre-Pregnancy Body Mass Index with Breastfeeding Initiation. Matern Child Health J. 2013, 17, 1842-1851. [CrossRef] [PubMed]

47. Amir, L.H.; Donath, S. A Systematic Review of Maternal Obesity and Breastfeeding Intention, Initiation and Duration. BMC Pregnancy Childbirth 2007, 7, 9. [CrossRef] [PubMed]

48. Bever Babendure, J.; Reifsnider, E.; Mendias, E.; Moramarco, M.W.; Davila, Y.R. Reduced Breastfeeding Rates among Obese Mothers: A Review of Contributing Factors, Clinical Considerations and Future Directions. Int. Breastfeed. J. 2015, 10, 21. [CrossRef] [PubMed]

49. Chapman, D.J.; Pérez-escamilla, R. Identification of Risk Factors for Delayed Onset of Lactation. J. Am. Dietetic Assoc. 1999, 99, 450-454. [CrossRef]

50. Lovelady, C.A. Is Maternal Obesity a Cause of Poor Lactation Performance? Nutr. Rev. 2005, 63, 352-355. [CrossRef] [PubMed]

51. Robinson, D.T.; Josefson, J.; Van Horn, L. Considerations for Preterm Human Milk Feedings When Caring for Mothers Who Are Overweight or Obese. Adv. Neonatal Care 2019, 19, 361-370. [CrossRef] [PubMed]

52. Knight, C.H. An Endocrine Hypothesis to Explain Obesity-Related Lactation Insufficiency in Breastfeeding Mothers. J. Dairy Res. 2020, 87, 78-81. [CrossRef]

53. Lei, W.-T.; Huang, K.-Y.; Jhong, J.-H.; Chen, C.-H.; Weng, S.-L. Metagenomic Analysis of the Gut Microbiome Composition Associated with Vitamin D Supplementation in Taiwanese Infants. Sci. Rep. 2021, 11, 2856. [CrossRef]

54. Li, Y.C.; Chen, Y.; Du, J. Critical Roles of Intestinal Epithelial Vitamin D Receptor Signaling in Controlling Gut Mucosal Inflammation. J. Steroid Biochem. Mol. Biol. 2015, 148, 179-183. [CrossRef] [PubMed]

55. Kanhere, M.; Chassaing, B.; Gewirtz, A.T.; Tangpricha, V. Role of Vitamin D on Gut Microbiota in Cystic Fibrosis. J. Steroid Biochem. Mol. Biol. 2018, 175, 82-87. [CrossRef]

56. Liu, W.; Chen, Y.; Golan, M.A.; Annunziata, M.L.; Du, J.; Dougherty, U.; Kong, J.; Musch, M.; Huang, Y.; Pekow, J.; et al. Intestinal Epithelial Vitamin D Receptor Signaling Inhibits Experimental Colitis. J. Clin. Investig. 2013, 123, 3983-3996. [CrossRef]

57. Prior, E.; Santhakumaran, S.; Gale, C.; Philipps, L.H.; Modi, N.; Hyde, M.J. Breastfeeding after Cesarean Delivery: A Systematic Review and Meta-Analysis of World Literature. Am. J. Clin. Nutr. 2012, 95, 1113-1135. [CrossRef]

58. Hermansson, H.; Kumar, H.; Collado, M.C.; Salminen, S.; Isolauri, E.; Rautava, S. Breast Milk Microbiota Is Shaped by Mode of Delivery and Intrapartum Antibiotic Exposure. Front. Nutr. 2019, 6, 4. [CrossRef]

59. Rautava, S.; Luoto, R.; Salminen, S.; Isolauri, E. Microbial Contact during Pregnancy, Intestinal Colonization and Human Disease Nat. Rev. Gastroenterol. Hepatol. 2012, 9, 565-576. [CrossRef]

60. Wachs, T.D.; Creed-Kanashiro, H.; Cueto, S.; Jacoby, E. Maternal Education and Intelligence Predict Offspring Diet and Nutritional Status. J. Nutr. 2005, 135, 2179-2186. [CrossRef]

61. Turroni, F.; Milani, C.; Duranti, S.; Mancabelli, L.; Mangifesta, M.; Viappiani, A.; Lugli, G.A.; Ferrario, C.; Gioiosa, L.; Ferrarini, A.; et al. Deciphering Bifidobacterial-Mediated Metabolic Interactions and Their Impact on Gut Microbiota by a Multi-Omics Approach. ISME J. 2016, 10, 1656-1668. [CrossRef]

62. Okada, Y.; Tsuzuki, Y.; Hokari, R.; Komoto, S.; Kurihara, C.; Kawaguchi, A.; Nagao, S.; Miura, S. Anti-Inflammatory Effects of the Genus Bifidobacterium on Macrophages by Modification of Phospho-IкB and SOCS Gene Expression. Int. J. Exp. Pathol. 2009, 90, 131-140. [CrossRef]

63. Baumann-Dudenhoeffer, A.M.; D'Souza, A.W.; Tarr, P.I.; Warner, B.B.; Dantas, G. Infant Diet and Maternal Gestational Weight Gain Predict Early Metabolic Maturation of Gut Microbiomes. Nat. Med. 2018, 24, 1822-1829. [CrossRef]

64. Chávez-Carbajal, A.; Nirmalkar, K.; Pérez-Lizaur, A.; Hernández-Quiroz, F.; Ramírez-del-Alto, S.; García-Mena, J.; HernándezGuerrero, C. Gut Microbiota and Predicted Metabolic Pathways in a Sample of Mexican Women Affected by Obesity and Obesity Plus Metabolic Syndrome. Int. J. Mol. Sci. 2019, 20, 438. [CrossRef] 
65. Kameyama, K.; Itoh, K. Intestinal Colonization by a Lachnospiraceae Bacterium Contributes to the Development of Diabetes in Obese Mice. Microbes Environ. 2014, 29, 427-430. [CrossRef]

66. Shen, F.; Zheng, R.-D.; Sun, X.-Q.; Ding, W.-J.; Wang, X.-Y.; Fan, J.-G. Gut Microbiota Dysbiosis in Patients with Non-Alcoholic Fatty Liver Disease. Hepatobiliary Pancreatic Diseases Int. 2017, 16, 375-381. [CrossRef]

67. Vacca, M.; Celano, G.; Calabrese, F.M.; Portincasa, P.; Gobbetti, M.; De Angelis, M. The Controversial Role of Human Gut Lachnospiraceae. Microorganisms 2020, 8, 573. [CrossRef]

68. Luthold, R.V.; Fernandes, G.R.; Franco-de-Moraes, A.C.; Folchetti, L.G.D.; Ferreira, S.R.G. Gut Microbiota Interactions with the Immunomodulatory Role of Vitamin D in Normal Individuals. Metabolism 2017, 69, 76-86. [CrossRef] [PubMed]

69. Waterhouse, M.; Hope, B.; Krause, L.; Morrison, M.; Protani, M.M.; Zakrzewski, M.; Neale, R.E. Vitamin D and the Gut Microbiome: A Systematic Review of in Vivo Studies. Eur. J. Nutr. 2019, 58, 2895-2910. [CrossRef]

70. Yassour, M.; Vatanen, T.; Siljander, H.; Hämäläinen, A.-M.; Härkönen, T.; Ryhänen, S.J.; Franzosa, E.A.; Vlamakis, H.; Huttenhower, C.; Gevers, D.; et al. Natural History of the Infant Gut Microbiome and Impact of Antibiotic Treatments on Strain-Level Diversity and Stability. Sci. Transl. Med. 2016, 8, 343ra81. [CrossRef] [PubMed]

71. Liu, Y.; Qin, S.; Song, Y.; Feng, Y.; Lv, N.; Xue, Y.; Liu, F.; Wang, S.; Zhu, B.; Ma, J.; et al. The Perturbation of Infant Gut Microbiota Caused by Cesarean Delivery Is Partially Restored by Exclusive Breastfeeding. Front. Microbiol. 2019, 10, 598. [CrossRef] [PubMed] 